\title{
Outcome dependent growth curves for singleton pregnancies based on birth weight of babies for Polish population
}

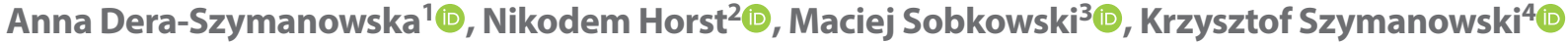 \\ ${ }^{1}$ Department of Perinatology and Gynecology, Poznan University of Medical Sciences, Poznan, Poland \\ ${ }^{2}$ Department of General and Colorectal Surgery, Poznan University of Medical Sciences, Poznan, Poland \\ ${ }^{3}$ Department of Mother's and Child's Health, Poznan University of Medical Sciences, Poznan, Poland \\ ${ }^{4}$ Department of Medical Education, Poznan University of Medical Sciences, Poznan, Poland
}

\begin{abstract}
Objectives: To create outcome dependent fetal growth curves and birth weight standards that can be analyzed for use in clinic specifically for Western European populations.

Material and methods: We conducted a retrospective study on fetal growth and birth weight trends from live birth singleton pregnancies between 2005 and 2018 at one of the largest tertiary gynecologic-obstetric hospitals in Poland. The inclusion criteria were at least 22 weeks of gestation at birth regardless of delivery mode (vaginal or C-section), no congenital anomalies diagnosed before and after delivery and an Apgar score of at least 7 in the first minute. The final sample had a total of 39,413 cases $\left(18,562\right.$ girls and 20,851 boys). We presented 7 (for all fetuses in the $5^{\text {th }}, 10^{\text {th }}, 25^{\text {th }}, 50^{\text {th }}, 75^{\text {th }}, 90^{\text {th }}$ and $95^{\text {th }}$ percentiles) and 6 (for boys and girls each at $10^{\text {th }}, 50^{\text {th }}$ and $90^{\text {th }}$ percentiles) fetal growth curves between 25 and 40 weeks of gestation. Birth weight trends were obtained and analyzed from all babies in the $5^{\text {th }}, 10^{\text {th }}, 25^{\text {th }}, 50^{\text {th }}, 75^{\text {th }}$ and $95^{\text {th }}$ percentiles born between 22 to 42 weeks of gestation with also separate trends for boys and girls.

Results: The largest differences in fetal growth curves were observed in the $10^{\text {th }}$ and $50^{\text {th }}$ percentiles between 22 and 34 weeks of gestation. A decreasing fetal weight gain pattern was observed between 27 and 30 weeks and after 38 weeks of gestation, the decrease was more drastic in female. A significant increase from 2009 to 2017 was observed in the weight of $50^{\text {th }}$ percentile babies born at or after 35 weeks. We found significant discrepancies between our results and the most used European fetal growth curves particularly in the $10^{\text {th }}$ and $90^{\text {th }}$ percentile weights at 30 weeks.

Conclusions: Separate scales for boys and girls were implied and given the overall difference form commonly used references. We believe there is significant value in using these unique patterns found in fetal growth curves and birth weights of ethnically homogenous population (such as Poland) at everyday clinical practice for more opportunities of safe obstetric care and higher chances of delivering a healthy child.
\end{abstract}

Key words: outcome dependent growth curves, singleton pregnancy

Ginekologia Polska 2020; 91, 12: 740-747

\section{INTRODUCTION}

In everyday clinical practice, we make extremely important decisions regarding the continuation of pregnancy or delivery. In our decisions, most often we rely on the gestational age and biophysical assessment of the fetus. Sometimes they result from the analysis of biochemical or hematological results concerning both the mother and the fetus. However, the estimated weight of the fetus and its implied maturity play a key role. If we are dealing with fetal growth disorders, the reference of the week of pregnancy, confirmed in the first trimester of pregnancy, to that de- termined based on ultrasound imaging, is one of the most important factors in our analysis. In pregnancy after the $34^{\text {th }}$ week, decisions are much easier, but when faced with prematurity, with extremely low fetal weight, making such a decision is one of the most difficult challenges in obstetrics. We have various fetal growth curves included in the programs that support our ultrasound devices, but none of these scales is based on the Polish population. Each of them, based on the same fetal measurements, shows incoherent weeks of pregnancy as well as difference in estimated fetal weight [1-3]. Not without significance are differences in the 
body proportions between individual races and different ways of creating growth curves.

There is no national birth registry in Poland, hence the creation of a full database is impossible. The last data showing the birth weight of the fetuses come from about 30 years ago, since then the living conditions have changed dramatically. All foreign, available databases contain an image of a very mixed racial population. In view of the very uniform nationality structure of our country, it seems necessary to create our own database.

The aim of this study was to create an outcome-dependent growth curves and birth weight standards for Polish population, basing on database of biggest tertiary care hospital in Poland.

\section{MATERIAL AND METHODS}

Data for the study were retrieved from the database of a tertiary care woman hospital in western Poland, for all patients that delivered between 01.01.2005 and 31.03.2018. Both the patients that electively chose our center as a first line care as well as the patients transferred from the other hospitals, including primary and secondary care, were included in the study.

The database included: date of birth, gestational age (completed weeks of pregnancy), sex, birth weight (rounded to $10 \mathrm{~g}$ ), mother's parity and age, mode of delivery and Apgar scores at 1, 3, 5 and 10 minutes. The database did not have information about pre and postnatal care, maternal health condition and whether the pregnancy resulted from assisted reproduction techniques.

The gestational age was based on LMP and was confirmed on ultrasound examination at the first trimester of pregnancy. The weight of each child was acquired by qualified personnel on electronic scale after calibration.

Inclusion criteria for the study were: (i) Babies born from singleton pregnancies at least in $22^{\text {nd }}$ gestational week without regard to the way of delivery (natural vs. C-section); (ii) No congenital anomalies.

Because this study was intended to be outcome-dependent and the database did not yield any information about postnatal course, all children that might have had high risk of unfavorable outcome had to be identified. Thus, following exclusion criteria was used: an Apgar scored in the first minute less than 7 or deteriorating in consecutive measurements $[4,5]$.

Due to the statistical nature of this study we identified and excluded outliers from the sample. Because the fetal mass within each gestational age did not have the normal distribution, we chose to identify the outliers without any method referring to a standard deviation. Since all the data were acquired by trained personnel, we decided to remove only extreme outliers within each gestational week, after applying all fore mentioned criteria. All fetuses within gestational week that had a mass below the $3^{\text {rd }}$ or above the $97^{\text {th }}$ percentile was identified as extreme outliers, which were $0.5 \%$ of the cases.

After applying all criteria, 2769 out of 42,182 records were excluded. The final sample had 39,413 cases (18,562 girls and 20,851 boys).

\section{Statistical analysis}

The Shaphiro-Wilk test revealed a non-normal distribution of weight and sex regarding the week of gestation. To alleviate this, we used the Generalized Additive Model for Location Scale and Shape (GAMLSS) which has been applied for data that has lost normality, for example when the distributions are skewed or kurtotic. This non-linear model was used to create growth curves by the World Health Organization [6, 7].

Prior to using GAMLSS, the distribution and smoothing method for tested groups were applied by fitting all relevant distributions and choosing the one fitting the best. Correctness was checked by visual inspection of theoretical and calculated percentiles and worm plots regarding gestational age.

All the calculations were performed in Microsoft Windows, with GAMLSS package ver. 5.0-6 for R ver. 3.4.3 in RStudio ver. 1.1.419 framework. Methods are described in our manuscript "Growth curves for twins for polish population" - in press).

\section{RESULTS}

According to the statistical nature of the study all results are presented on histograms and tables. On the five histograms we have shown distribution of the groups according to the week of delivery (Fig. 1), sex of the baby in consecutive weeks (Fig. 2), fetal mass according to week of delivery (Figs. 3 and 4) and finally centile curves using Box-Cox Power Exponential for the entire study group. Table 1 presents percentiles of the fetal birth weight for the entire study group. Distribution of the centiles separately for girls and boys are presented on Figure 5 and in Tables 2 and 3. Table 4 was created to show annual changes.

\section{DISCUSSION}

Seventy percent of infants born below the $10^{\text {th }}$ percentile are not at risk for adverse outcomes [8]. Therefore, we search for this $30 \%$ of babies with growth restriction and severe risk of adverse outcome. The Society of Obstetricians and Gynecologists of Canada (SGOC) and the Royal College of Obstetricians and Gynecologists (RCOG) define 


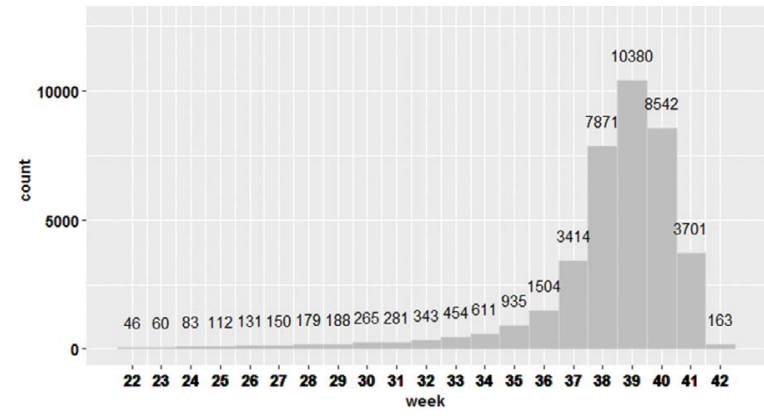

Figure 1. Histogram of the final group according to the week of delivery

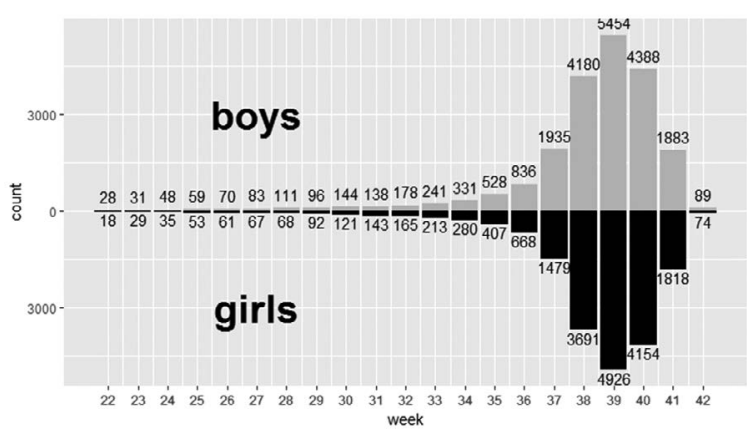

Figure 2. Histogram of the final group according to the sex of the babies and completed weeks at the delivery

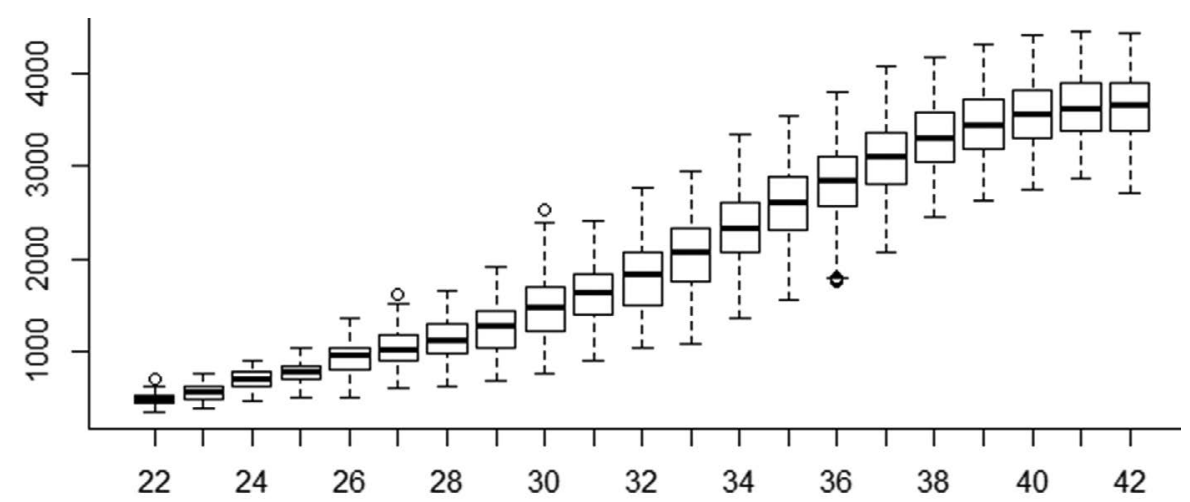

Figure 3. Distribution of the fetal mass after delivery when estimated rough to completed weeks

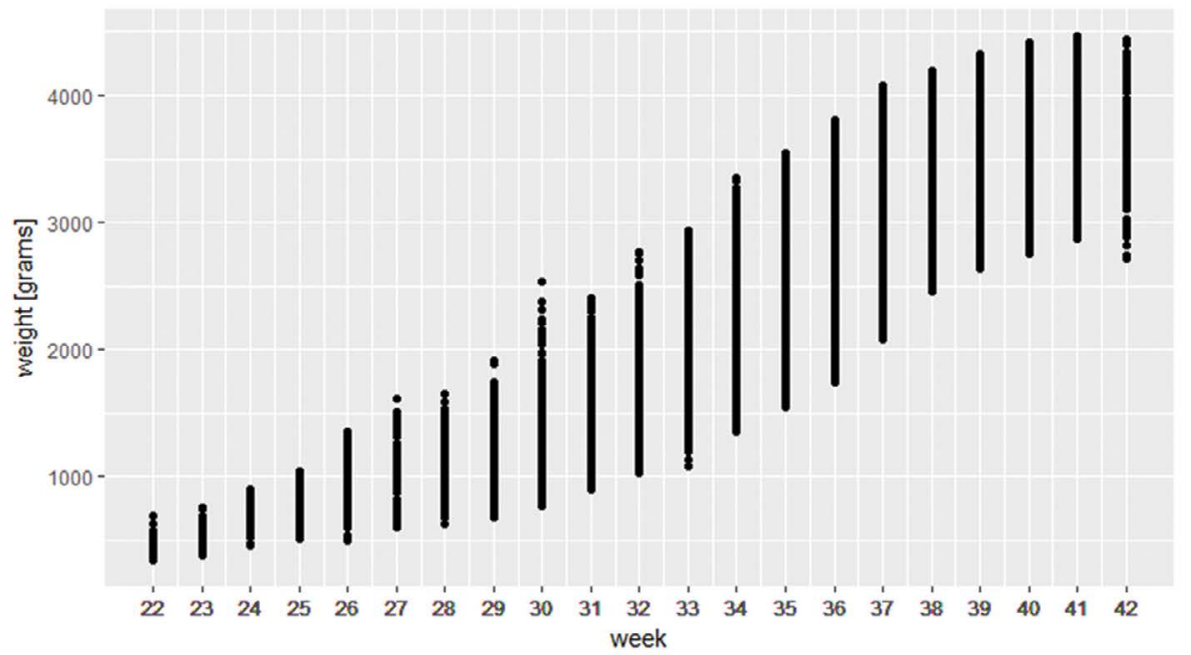

Figure 4. Distribution of the fetal mass after delivery when estimated rough to completed weeks

fetal growth restriction (FGR) as an estimated fetal weight of $<10^{\text {th }}$ percentile on ultrasound in a fetus that, because of a pathologic process, has not attained its biologically determined growth potential [9]. American College of
Obstetricians and Gynecologists (ACOG) recommends "If the ultrasonographically estimated fetal weight is below the $10^{\text {th }}$ percentile for gestational age, further evaluation should be considered, such as amniotic fluid assessment 


\begin{tabular}{|c|c|c|c|c|c|c|c|}
\hline \multirow{2}{*}{ Gestational age [week] } & \multicolumn{7}{|c|}{ Percentiles } \\
\hline & C5 & C10 & C25 & C50 & C75 & C90 & C95 \\
\hline 22 & 368 & 395 & 440 & 485 & 532 & 578 & 607 \\
\hline 23 & 436 & 464 & 515 & 577 & 639 & 690 & 718 \\
\hline 24 & 508 & 542 & 604 & 682 & 758 & 817 & 849 \\
\hline 25 & 572 & 619 & 700 & 796 & 890 & 966 & 1008 \\
\hline 26 & 623 & 687 & 794 & 915 & 1035 & 1136 & 1194 \\
\hline 27 & 674 & 746 & 873 & 1026 & 1177 & 1300 & 1368 \\
\hline 28 & 726 & 803 & 946 & 1130 & 1310 & 1445 & 1515 \\
\hline 29 & 800 & 886 & 1047 & 1255 & 1463 & 1622 & 1707 \\
\hline 30 & 923 & 1018 & 1194 & 1416 & 1649 & 1847 & 1961 \\
\hline 31 & 1062 & 1164 & 1355 & 1599 & 1855 & 2070 & 2193 \\
\hline 32 & 1207 & 1319 & 1530 & 1807 & 2086 & 2304 & 2420 \\
\hline 33 & 1392 & 1521 & 1757 & 2053 & 2342 & 2562 & 2679 \\
\hline 34 & 1692 & 1779 & 2037 & 2335 & 2622 & 2854 & 2983 \\
\hline 35 & 1867 & 2032 & 2305 & 2607 & 2894 & 3129 & 3262 \\
\hline 36 & 2107 & 2282 & 2560 & 2850 & 3126 & 3364 & 3502 \\
\hline 37 & 2412 & 2560 & 2811 & 3098 & 3379 & 3615 & 3750 \\
\hline 38 & 2714 & 2824 & 3033 & 3308 & 3590 & 3813 & 3933 \\
\hline 39 & 2868 & 2971 & 3173 & 3448 & 3732 & 3953 & 4071 \\
\hline 40 & 2984 & 3086 & 3286 & 3559 & 3841 & 4057 & 4172 \\
\hline 41 & 3082 & 3181 & 3375 & 3642 & 3917 & 4130 & 4242 \\
\hline 42 & 3090 & 3203 & 3408 & 3666 & 3930 & 4147 & 4270 \\
\hline
\end{tabular}

A

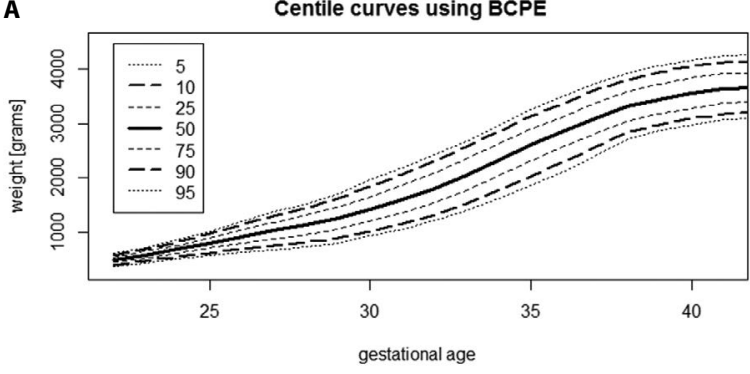

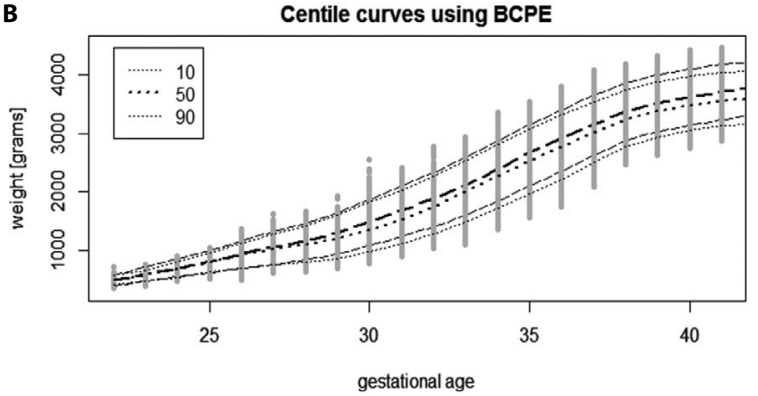

Figure 5. A. Centile curves for the entire study group; B. Centile curves separately for the boys and girls); BCPE — Box-Cox Power Exponential

and Doppler blood flow studies of the umbilical artery." [10]. In all guidelines, detection of the fetuses with estimated fetal weight below $10^{\text {th }}$ percentile is the first step. Further, searching for Doppler abnormalities should be performed in the carefully selected group of fetuses. It is worth mentioning a publication by Gordijn et al. [11] which presented a more precise expert consensus definition of fetal growth restriction. The experts agreed upon between early and late FGR (demarcation at 32 weeks of gestation), exclusion of congenital abnormalities, lower cut-offs size measurement of $<3^{\text {rd }}$ centile and functional parameters (solitary- absent end-diastolic flow in the UA, contributory UA-PI or Ut A-PI $>95^{\text {th }}$ centile or $\mathrm{CPR}<5^{\text {th }}$ centile). However, even for the group of true experts guided by Delphi procedure it is difficult to choose uniform and simple definition of fetal growth restriction. On the other 
Table 2. Percentiles of fetal birth weight with regard to the week of gestation for girls

\begin{tabular}{|c|c|c|c|c|c|c|c|}
\hline \multirow{2}{*}{ Gestational age [week] } & \multicolumn{7}{|c|}{ Percentiles } \\
\hline & C5 & C10 & C25 & C50 & C75 & C90 & C95 \\
\hline 22 & 372 & 398 & 440 & 484 & 526 & 565 & 588 \\
\hline 23 & 428 & 457 & 506 & 559 & 612 & 659 & 686 \\
\hline 24 & 499 & 534 & 593 & 660 & 727 & 786 & 821 \\
\hline 25 & 575 & 619 & 695 & 781 & 868 & 945 & 992 \\
\hline 26 & 641 & 697 & 793 & 905 & 1019 & 1121 & 1181 \\
\hline 27 & 681 & 747 & 866 & 1007 & 1152 & 1277 & 1349 \\
\hline 28 & 709 & 785 & 922 & 1095 & 1270 & 1416 & 1499 \\
\hline 29 & 778 & 855 & 1003 & 1200 & 1412 & 1591 & 1694 \\
\hline 30 & 900 & 976 & 1128 & 1345 & 1594 & 1817 & 1950 \\
\hline 31 & 1027 & 1112 & 1282 & 1524 & 1797 & 2033 & 2169 \\
\hline 32 & 1170 & 1271 & 1470 & 1743 & 2033 & 2265 & 2391 \\
\hline 33 & 1345 & 1468 & 1700 & 1999 & 2298 & 2528 & 2651 \\
\hline 34 & 1574 & 1713 & 1963 & 2270 & 2575 & 2819 & 2953 \\
\hline 35 & 1804 & 1958 & 2220 & 2522 & 2821 & 3075 & 3221 \\
\hline 36 & 2055 & 2215 & 2480 & 2771 & 3057 & 3310 & 3459 \\
\hline 37 & 2368 & 2504 & 2741 & 3019 & 3300 & 3544 & 3687 \\
\hline 38 & 2671 & 2773 & 2968 & 3229 & 3509 & 3744 & 3878 \\
\hline 39 & 2838 & 2932 & 3118 & 3376 & 3654 & 3883 & 4010 \\
\hline 40 & 2951 & 3046 & 3231 & 3486 & 3759 & 3981 & 4103 \\
\hline 41 & 3040 & 3133 & 3313 & 3560 & 3826 & 4047 & 4171 \\
\hline 42 & 3047 & 3151 & 3345 & 3592 & 3850 & 4066 & 4189 \\
\hline
\end{tabular}

hand, clinically relevant is the determination of children over 90 centiles in terms of perinatal complications.

We are aware of all the inaccuracies that ultrasound carries - overestimation in large fetuses and underestimation of small fetuses, differences between races, and most often - human error. However, we believe that the determination of the fetal weight gain for individual weeks of pregnancy should be constructed based on the population in which we operate and, on the births, already performed. How can we know the actual weight of the fetus by ultrasound if errors cannot be eliminated? In the opposite, Lackman et al. [12], have advocated that "...intrauterine growth curves derived from ultrasonographically estimated fetal weight of continuing pregnancies are more appropriate than neonatal growth curves to discriminate fetuses and neonates at higher risk for adverse outcome."

In our opinion, the weight of newborns is the only objective assessment method. However, in order to create growth curves reflecting the undisturbed development of children with different growth curves, we rejected extreme outliers and children with: an Apgar scored in the first minute less than 7 or deteriorating in consecutive measurements. There is also a risk of incorrect selection of fetuses born in extremely low weeks. How often does a child born in 22 weeks have Apgar 7 or more?

Another necessary element is separate analysis of fetuses of different sexes. The Polish population is very homogeneous, hence there is no need for separate analysis of individual race. This need is indicated, for example, by the data provided by the National Institute of Child Health and Human Development (NICHD) Fetal Growth Studies which demonstrates the differences in fetal growth between different races and ethnicities [13]. These differences concern both $10^{\text {th }}$ and $90^{\text {th }}$ percentile. For example, according to Hadlock et al. [1] the $10^{\text {th }}$ percentile for $30^{\text {th }}$ week is $1294 \mathrm{~g}$, according to Duryea et al. [14] - $1068 \mathrm{~g}$. We estimated this to be $1018 \mathrm{~g}$. Assessing gender separately, the value for girls is 976 , and for boys 1018 . In the higher weeks of pregnancy these proportions are reversed. For week 40 they are $3004 \mathrm{~g}$, $3005 \mathrm{~g}$ and $3086 \mathrm{~g}$, respectively. For the girls we achieved in our chart $3046 \mathrm{~g}$ and for the boys - $3138 \mathrm{~g}$. Comparing $90^{\text {th }}$ centile for $40^{\text {th }}$ week the differences seem high. Thus, 
Table 3. Percentiles of fetal birth weight with regard to the week of gestation for boys

\begin{tabular}{|c|c|c|c|c|c|c|c|}
\hline \multirow{2}{*}{ Gestational age [week] } & \multicolumn{7}{|c|}{ Percentiles } \\
\hline & C5 & C10 & C25 & C50 & C75 & C90 & C95 \\
\hline 22 & 368 & 390 & 430 & 480 & 532 & 576 & 602 \\
\hline 23 & 441 & 471 & 524 & 590 & 656 & 710 & 740 \\
\hline 24 & 504 & 543 & 613 & 697 & 778 & 843 & 878 \\
\hline 25 & 560 & 611 & 701 & 806 & 907 & 985 & 1027 \\
\hline 26 & 620 & 686 & 800 & 933 & 1059 & 1156 & 1208 \\
\hline 27 & 678 & 756 & 892 & 1051 & 1201 & 1317 & 1378 \\
\hline 28 & 738 & 825 & 977 & 1155 & 1325 & 1455 & 1526 \\
\hline 29 & 831 & 928 & 1096 & 1294 & 1485 & 1634 & 1715 \\
\hline 30 & 964 & 1071 & 1257 & 1478 & 1694 & 1865 & 1959 \\
\hline 31 & 1111 & 1229 & 1433 & 1674 & 1909 & 2099 & 2204 \\
\hline 32 & 1266 & 1396 & 1618 & 1877 & 2128 & 2331 & 2444 \\
\hline 33 & 1449 & 1595 & 1838 & 2112 & 2374 & 2588 & 2707 \\
\hline 34 & 1672 & 1835 & 2099 & 2382 & 2649 & 2871 & 2997 \\
\hline 35 & 1920 & 2097 & 2374 & 2661 & 2929 & 3154 & 3283 \\
\hline 36 & 2183 & 2357 & 2631 & 2917 & 3183 & 3407 & 3535 \\
\hline 37 & 2482 & 2631 & 2881 & 3164 & 3434 & 3654 & 3777 \\
\hline 38 & 2751 & 2875 & 3097 & 3373 & 3644 & 3855 & 3969 \\
\hline 39 & 2912 & 3025 & 3239 & 3517 & 3792 & 4000 & 4109 \\
\hline 40 & 3029 & 3138 & 3349 & 3629 & 3906 & 4111 & 4216 \\
\hline 41 & 3134 & 3239 & 3444 & 3719 & 3990 & 4187 & 4287 \\
\hline 42 & 3213 & 3316 & 3514 & 3781 & 4042 & 4231 & 4326 \\
\hline
\end{tabular}

Hadlock et al. [1] estimated this for $4234 \mathrm{~g}$, Duryea et al. [14] - $4057 \mathrm{~g}$. We have obtained the same value as Duyrea et al. [14] - $4057 \mathrm{~g}$. However, comparing the girls and boys we have $3981 \mathrm{~g}$ and $4111 \mathrm{~g}$, respectively.

It is not difficult to notice differences reaching almost $250 \mathrm{~g}$ depending on the evaluated curves, as well as comparing them shows quite different fetal growth curves. Therefore, is it possible to make key decisions for obstetricians in children with Small for Gestational Age (SGA) or with macrosomia, without knowing what the correct fetal mass is for a given population? It was stressed already in data from the Global Survey which showed that birthweight at 40 completed weeks of gestation varied between $2790 \mathrm{~g}$ in India and $3511 \mathrm{~g}$ in Algeria, which is well below the mean birthweight for the women from the European Continental Ancestry Group in the original study sample used to develop the ultrasound reference by Hadlock et al. [15]. An interesting study was presented by Nicolaides et al. [16], where reference population for birth weight charts were derived from all babies - born and those still in utero.
They assumed that median for birth weight is the same as estimated fetal weight done shortly before birth. They believe strongly that the sonographic estimation is fully accurate. Is it true? This is what we are afraid of. As for us, the underlined strength of the study seems its weakness. The other study - INTERGROWTH $21^{\text {st }}$ project [17] encompasses population from 8 countries from 5 different continents. The authors applied many exclusion criteria: maternal age younger than 18 or over 35 , maternal height shorter than 153 , body mass index $\geq 30 \mathrm{~kg} / \mathrm{m}^{2}$ or $\leq 18.5 \mathrm{~kg} / \mathrm{m}^{2}$, current smoker, medical history, birth of any previous baby weighing less than $2.5 \mathrm{~kg}$ or more than $4.5 \mathrm{~kg}$, past 2 pregnancies ending in miscarriage, any previous stillbirth or neonatal death, or congenital malformation. However, if the week of pregnancy was allowed to be based on biparietal diameter done before 24 weeks and most of the living condition are completely different between each other in aspects of geography, ethnicity and most of all life standard, we have some doubts concerning usefulness of such birth charts. 


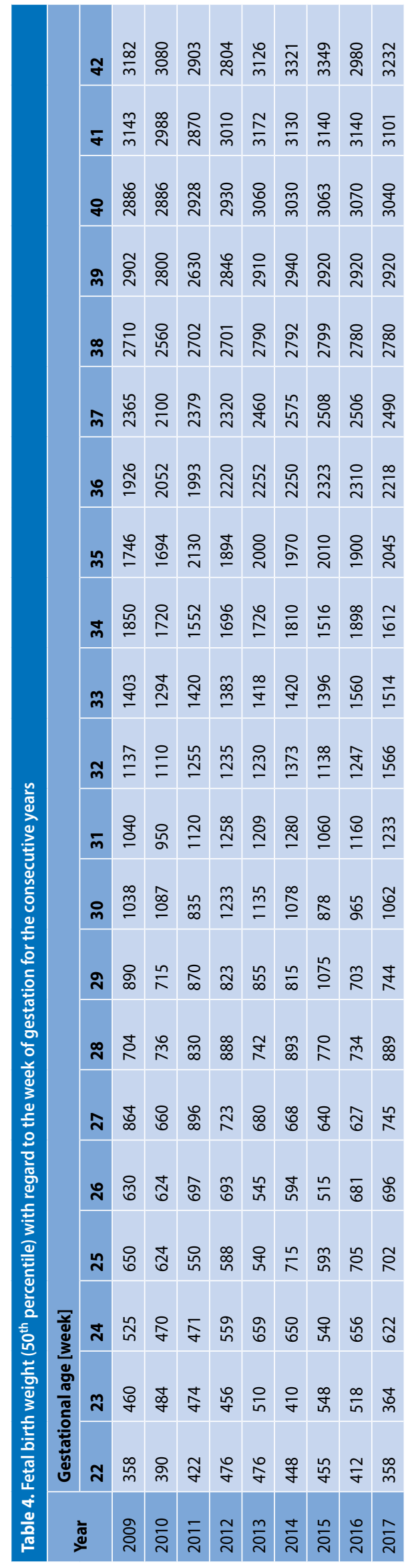

Analyzing the birth curves, we obtained, it seems extremely important to analyze them weekly. Comparison of the $10^{\text {th }}$ percentile and 50 percentiles for both boys and girls shows some analogies. The largest differences in growth curves (centiles $10^{\text {th }} \mathrm{vs.} 50^{\text {th }}$ ) were obtained between weeks 22 and 34 . After this time, the fetuses from the $10^{\text {th }}$ percentile compared to the fetuses from the $50^{\text {th }}$ percentile show a larger mass gain. It can be assumed that if there was an earlier delivery due to Intrauterine Growth Restriction (IUGR) or other underlying pathologies, then hypotrophic fetuses tend to make up for the mass required.

It can therefore be assumed that pregnancy is relatively safe during this period. Our previous feelings seemed different. The above-described group of hypotrophic fetuses with accelerated growth after 35 weeks may be just a group of healthy fetuses with constitutionally lower weight.

It is worth emphasizing the slowing down of weight gain for both sexes and for both percentiles analyzed above between 27 and 30 weeks and after 38 weeks of pregnancy. If the correct weight gain for the $10^{\text {th }}$ percentile at week 33 is about $200 \mathrm{~g}$ for girls and boys, between 34 and 37 is for both sexes and centiles between 240 and $289 \mathrm{~g}$, then for the following weeks it is about $150 \mathrm{~g}$ at week 39 , in 40 - around 115, and in the following weeks it decreases drastically, especially for female fetuses. The above data indicate the need to create customized fetal growth charts with separate scales for boys and girls.

The improvement of living conditions, or maybe differently - the improvement of society's health, and at the same time a change in eating habits can have a significant impact on accelerating the secular trend in the birth weight of newborns. An analysis of our material, where we compared 50 percentiles over the 9 years studied, indicates a slight increase in newborns mass, especially those born after 35 weeks of pregnancy (Table 4).

The overriding goal of our research is to determine the birth curve of fetal masses that can be used in everyday clinical work. By rejecting extreme outliers and children born in moderate or poor condition, we excluded a significant proportion of obstetric pathologies that could have a significant effect on fetal weight. Despite the methodological conditions and imperfections in the construction of each of the scales, we have proposed one that we believe will allow us to safely conduct obstetric supervision, giving the best chance of giving birth to a healthy child.

When assessing the weight of the fetus, and at the same time maintaining common sense, we should have in mind the ACOG hint on the assessment of fetal weight in macrosomia. "An accurate diagnosis of macrosomia can be made only by weighing the newborn after delivery." [18]. 


\section{CONCLUSIONS}

Week-to-week weight gain equal to or higher than $200 \mathrm{~g}$ at week 33 and minimum $240 \mathrm{~g}$ between 34 and 37 weeks seems to be good predictor of favorable outcome in absence of the other pathologies for both sexes.

\section{REFERENCES}

1. Hadlock FP, Harrist RB, Martinez-Poyer J. In utero analysis of fetal growth: a sonographic weight standard. Radiology. 1991; 181(1): 129-133, doi: 10.1148/radiology.181.1.1887021, indexed in Pubmed: 1887021.

2. Committee opinion no 611: method for estimating due date. Obstet Gynecol. 2014; 124(4): 863-866, doi: 10.1097/01.AOG.0000454932.15177. be, indexed in Pubmed: 25244460.

3. Butt K, Lim K, Lim K, et al. Determination of gestational age by ultrasound. J Obstet Gynaecol Can. 2014; 36(2): 171-181, doi: 10.1016/s17012163(15)30664-2.

4. Sørensen HT, Ehrenstein V, Pedersen L, et al. Association of Apgar score at five minutes with long-term neurologic disability and cognitive function in a prevalence study of Danish conscripts. BMC Pregnancy Childbirth. 2009: 9: 14, doi: 10.1186/1471-2393-9-14, indexed in Pubmed: 19341459.

5. Li F, Wu T, Lei $X$, et al. The apgar score and infant mortality. PLoS One. 2013; 8(7): e69072, doi: 10.1371/journal.pone.0069072, indexed in Pubmed: 23922681.

6. Borghi E, de Onis M, Garza C, et al. WHO Multicentre Growth Reference Study Group. Construction of the World Health Organization child growth standards: selection of methods for attained growth curves. Stat Med. 2006; 25(2): 247-265. Erratum in: Stat Med. 2008; 27(16): 3216, doi: 10.1002/sim.2227, indexed in Pubmed: 16143968.

7. Ramos $F$, Pérez $G$, Jané $M$, et al. Construction of the birth weight by gestational age population reference curves of Catalonia (Spain): Methods and development. Gac Sanit. 2009; 23(1): 76-81, doi: 10.1016/j. gaceta.2008.03.001, indexed in Pubmed: 19231729.

8. Ott WJ.The diagnosis of altered fetal growth. Obstet Gynecol Clin North Am. 1988; 15(2): 237-263, indexed in Pubmed: 3067166.
9. Lausman A, Kingdom J, Gagnon R, et al. Intrauterine Growth Restriction: Screening, Diagnosis, and Management. J Obstet Gynaecol Can. 2013; 35(8): 741-748, doi: 10.1016/s1701-2163(15)30865-3.

10. Fetal growth restriction. Practice Bulletin No. 134. Obstetrics \& Gynecology. 2013; 121(5): 1122-1133, doi: 10.1097/01.aog.0000429658.85846.f9.

11. Gordijn SJ, Beune IM, Thilaganathan B, et al. Consensus definition of fetal growth restriction: a Delphi procedure. Ultrasound Obstet Gynecol. 2016; 48(3): 333-339, doi: 10.1002/uog.15884.

12. Lackman F, Capewell V, Richardson B, et al. The risks of spontaneous preterm delivery and perinatal mortality in relation to size at birth according to fetal versus neonatal growth standards. Am J Obstet Gynecol. 2001; 184(5): 946-953, doi: 10.1067/mob.2001.111719, indexed in Pubmed: 11303203.

13. Buck Louis GM, Grewal J, Albert PS, et al. Racial/ethnic standards for fetal growth: the NICHD Fetal Growth Studies. Am J Obstet Gynecol. 2015; 213(4): 449.e1-449.e41, doi: 10.1016/j.ajog.2015.08.032, indexed in Pubmed: 26410205.

14. Duryea EL, Hawkins JS, McIntire DD, et al. A revised birth weight reference for the United States. Obstet Gynecol. 2014; 124(1): 16-22, doi: 10.1097/AOG.0000000000000345, indexed in Pubmed: 24901276.

15. Mikolajczyk R, Zhang J, Betran A, et al. A global reference for fetal-weight and birthweight percentiles. Lancet. 2011; 377(9780): 1855-1861, doi: 10.1016/s0140-6736(11)60364-4.

16. Nicolaides $\mathrm{KH}$, Wright $\mathrm{D}$, Syngelaki A, et al. Fetal Medicine Foundation fetal and neonatal population weight charts. Ultrasound Obstet Gynecol. 2018; 52(1): 44-51, doi: 10.1002/uog.19073, indexed in Pubmed: 29696704.

17. Villar J, Cheikh Ismail L, Victora CG, et al. International Fetal and Newborn Growth Consortium for the 21st Century (INTERGROWTH-21st). International standards for newborn weight, length, and head circumference by gestational age and sex: the Newborn Cross-Sectional Study of the INTERGROWTH-21st Project. Lancet. 2014; 384(9946): 857-868, doi: 10.1016/S0140-6736(14)60932-6, indexed in Pubmed: 25209487.

18. Fetal macrosomia. Practice Bulletin No. 173. American College of Obstetricians and Gynecologists. Obstet Gynecol. 2016; 128: e195-209. 